\title{
In situ reconstruction of the external iliac artery and vein with autogenous vessels in a patient with rectosigmoid colon cancer invading the external iliac artery and vein: A case report
}

\author{
Young-Nam Roh, Pyong Wha Choi* \\ Department of Surgery, Ilsan Paik Hospital, Inje University College of Medicine, Goyang, Korea
}

Received: February 22, 2017

Accepted: April 6, 2017

Online Published: April 12, 2017

DOI: $10.5430 /$ css.v3n2p23

URL: https://doi.org/10.5430/css.v3n2p23

\begin{abstract}
Aggressive radical R0 resection is necessary for good prognosis in colorectal cancer with adjacent organ invasion. According to the location of the tumor, various pelvic organs are removed in en-block resection. However, en-block resection may be challenging in case with vascular invasion such as external iliac vessels because vascular specialist should be involved and vascular reconstruction is complex procedure than any other surgical procedure. We report the case of a 53-year-old man who underwent curative surgery for rectosigmoid colon cancer invading the left external iliac artery (EIA) and vein (EIV). En-bloc resection of the tumor including external iliac vessels and ureter was performed, and the resected EIA and EIV were reconstructed using the internal iliac artery and vein. This procedure is more physiologic than extra-anatomic bypass and eliminates the need of artificial graft. Consequently, its use may improve patency and decrease the risk of graft infection.
\end{abstract}

Key Words: Colon cancer, En-bloc resection, Vascular reconstruction, Autogenous vessel

\section{INTRODUCTION}

Vascular surgery must be performed in a clean operation field without bacterial contamination. A risk of graft infection is associated with the use of artificial grafts and can lead serious complications, including amputation and death. Therefore, vascular surgery is generally not performed adjunctive to clean-contaminated or contaminated surgery including colorectal surgery.

However, vascular reconstruction is often needed during colorectal surgery. Major vessel reconstruction in patients with cancer originating from the colon or rectum, where microorganisms of high virulence are always present, is especially challenging. In such cases, en-block resection including involved vessels combined with extra-anatomic bypass using an artificial graft is generally considered. ${ }^{[1-5]}$ However, if in situ reconstruction using an autogenous vessel is feasible, it may be more physiologic and can eliminate the risk of graft infection. We present our experience of successful in situ left external iliac artery (EIA) and vein (EIV) reconstruction without using an artificial graft during the radical resection of colorectal cancer invading the external iliac vessels.

\section{Case presentation}

A 53-year-old man presented with a 1-month history of abdominal pain and constipation. Abdomino-pelvic computed

\footnotetext{
*Correspondence: Pyong Wha Choi, MD, PhD; Email: choipeace1130@gmail.com; Address: Department of Surgery, Inje University College of Medicine, Ilsan Paik Hospital, 170, Juhwa-ro, Ilsanseo-gu, Goyang-si, Gyeonggi-do, 10380, Korea.
} 
tomography $(\mathrm{CT})$ revealed a rectosigmoid colon mass extending to the sigmoid mesocolon. The mass invaded the left mesorectal fascia abutting the left mid-ureter and left EIA. The proximal ureter was dilated, and the left external iliac vessels abutted the thickened mesocolon (see Figure 1). A sigmoidoscopy revealed a fungating mass in the sigmoid colon, and a biopsy revealed the presence of an adenocarcinoma. A positron emission tomography did not reveal any distant metastasis. We decided to perform radical resection; however, we were not sure whether the mass could be detached from the left EIA or not.

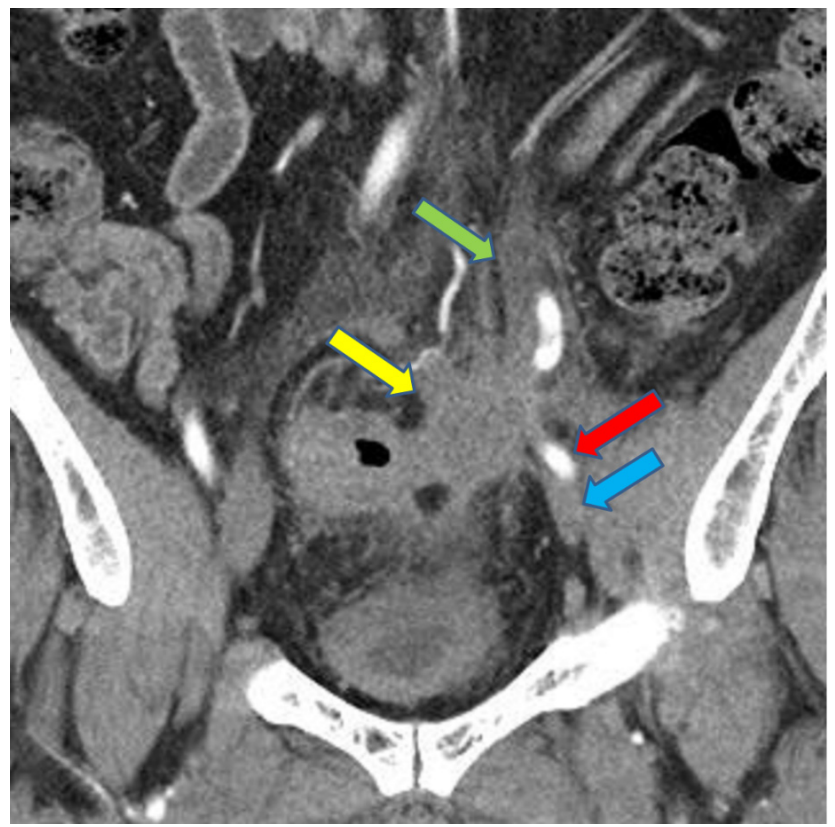

Figure 1. Rectosigmoid colon cancer invading the left EIA, EIV, and ureter on computerized tomography scan Yellow arrow, rectosigmoid colon cancer; Green arrow, dilated ureter; Red arrow, EIA; Blue arrow, EIV

During the operation, a low midline incision was made, and the sigmoid colon was mobilized. However, the mesocolon of the sigmoid colon was thickened and hard, and it was severely attached to the left EIA and EIV. On top of that, the ureter was firmly attached to the sigmoid mesocolon.

Since the patient was young and showed no evidence of distant metastasis, we decided to proceed with radical resection. The inferior mesenteric artery was ligated, and the proximal and distal portions of the EIA and EIV were encircled with a vessel loop. Then the EIA, EIV, and ureter were resected. Subsequently, the rectosigmoid colon was resected in a conventional manner. After the specimen was delivered (see Figure 2), a vascular surgeon reconstructed the resected iliac artery and vein. At first, EIA interposition with the artificial graft and simple ligation of the external iliac vein were con- sidered. However, we were not sure if the operative field had any bacterial contamination, because the colon mucosa was exposed during resection. Then we found that the internal iliac artery (IIA) and vein (IIV) were fully exposed after resection of the rectosigmoid colon; therefore, we decided to reconstruct the resected vessels with the IIA and IIV. The proximal end of the cut EIA was sutured, and then the IIA was mobilized distally until it divided into branches. This mobilized IIA was anastomosed to the distal EIA. The remnant proximal portion of the EIV was mobilized with the ligation of side branches, which connected to the distal EIV; however, it was not long enough for primary anastomosis. Therefore, a 3-cm segment of the IIV was harvested and interposed between the cut ends of the EIV (see Figure 3). Vascular anastomosis was performed with 6-0 prolene. The left ureter was anastomosed to the right ureter with end-toside anastomosis, and a double-J catheter was inserted. After vascular anastomosis, we performed anastomosis of colon to rectal stump followed diversion ileostomy. The total operation time was 5 hours 30 minutes and intraoperative blood loss was $780 \mathrm{ml}$. So, 2 units blood were transfused.

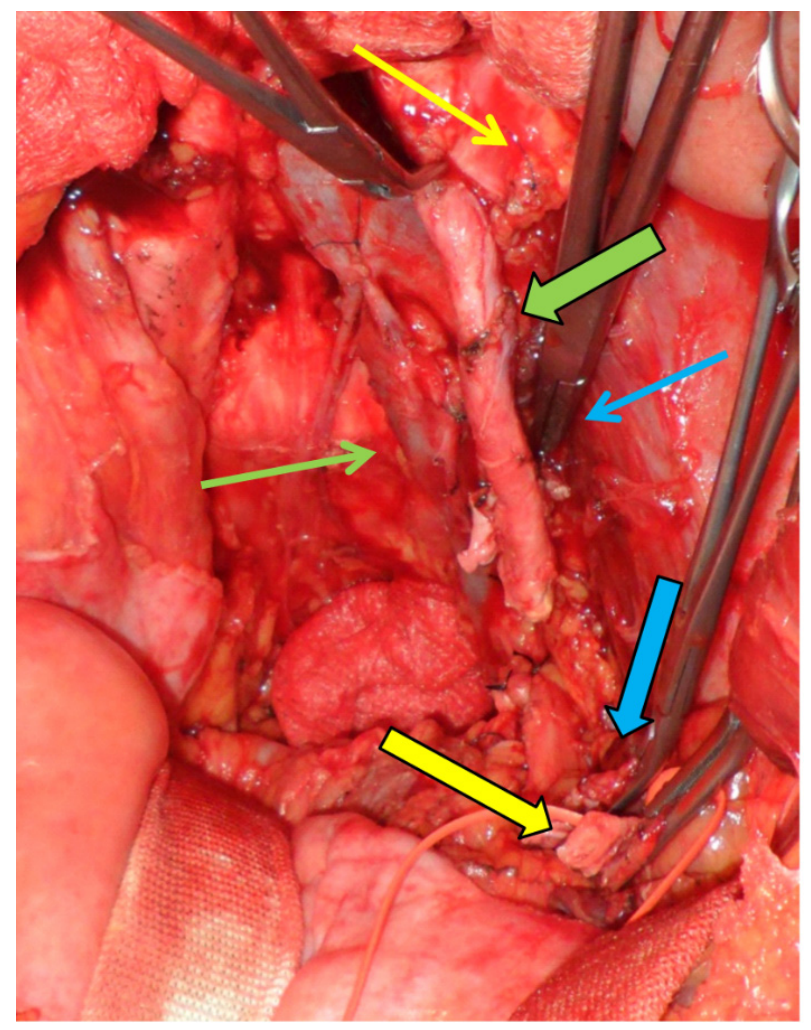

Figure 2. After en-bloc resection of colon cancer including the left EIA and EIV

Yellow thin arrow, sutured proximal EIA; Yellow thick arrow, clamped distal EIA; Blue thin arrow, clamped proximal EIV; Blue thick arrow, clamped distal EIV; Green thin arrow, IIV; Green thick arrow, mobilized IIA 


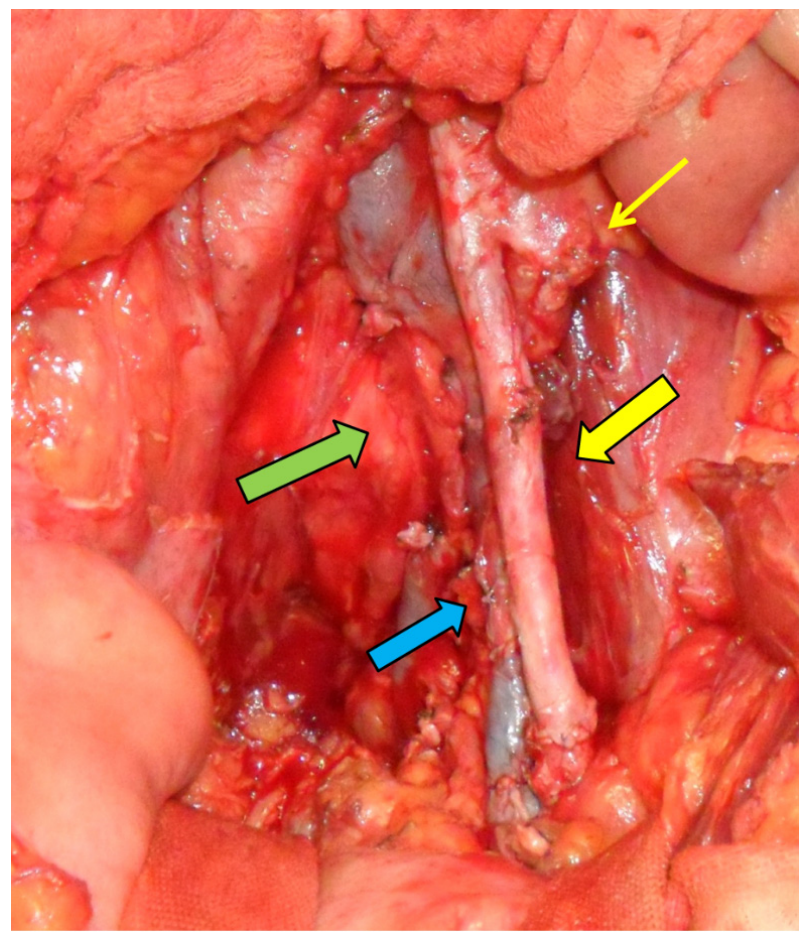

Figure 3. Transposition of the internal iliac artery with the EIA and EIV interposition with the IIV graft

Yellow thin arrow, sutured proximal EIA; Yellow thick arrow, transposition of the IIA with the EIA; Blue arrow, reconstructed EIV with interposition of the IIV graft; Green arrow, harvested segment of the IIV
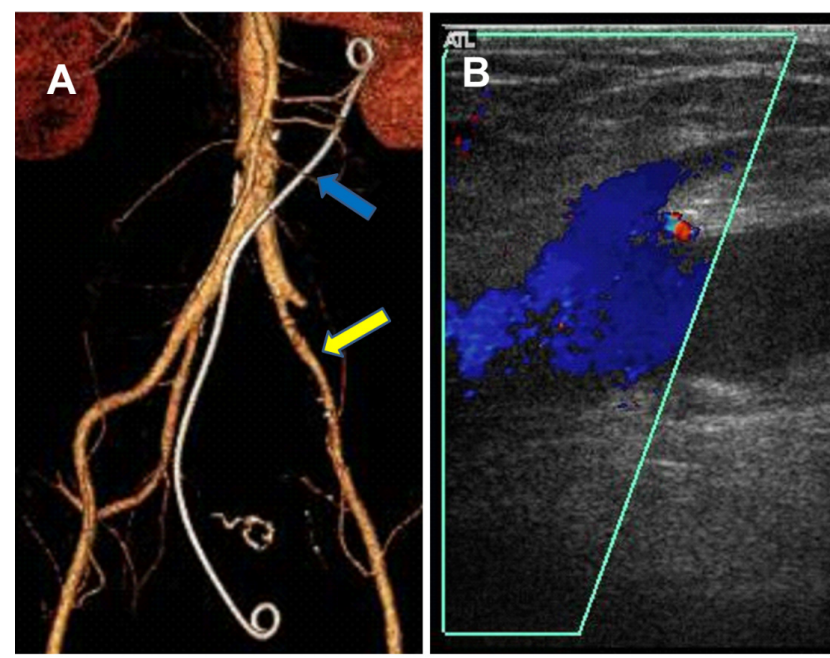

Figure 4. Follow-up imaging after the operation A.Transposed IIA (yellow arrow) on computerized tomography angiography (blue arrow, double J catheter) 1 month after the operation; B. Patent iliofemoral venous system on duplex scan 3 month after the operation

The postoperative course was uneventful, and anticoagulation was started on postoperative day 3 . But, gross hematuria was developed on postoperative $7^{\text {th }}$ day because of anticoPublished by Sciedu Press agulation and irritation from the double $\mathrm{J}$ stent. Observation and bed rest without any intervention could result in improving the symptom. Although the hospitalization period was extended due to recurrent gross hematuria, the patient was discharged on postoperative day 19 with anticoagulant medication. The patient was followed up for 3 months, and did not appeal claudication or swelling on the leg. Follow-up CT angiography on postoperative day 30 and duplex scan on postoperative day 90 revealed patent reconstructed artery and vein (see Figure 4). Ileostomy take down was performed 3 month after the surgery.

\section{Discussion}

Several reports on the reconstruction of major arteries during colorectal surgery concluded that aggressive expanded surgery can lead to R0 resection and improve oncologic outcomes. ${ }^{[1-5]}$ However, the details of vessel reconstruction were not thoroughly discussed and consensus was not achieved. Colorectal cancer often invades the iliac artery, but after resection of the involved iliac artery, obtaining autogenous graft material for arterial reconstruction is difficult because of the large caliber of the iliac artery. The alternative is using an artificial graft such as polytetrafluoroethylene (PTFE) or Dacron graft. However, vascular surgeons are reluctant to use artificial grafts in the field of colorectal surgery where there is possibility for bacterial contamination. There are several methods to avoid graft infection, such as omentum wrap around the artificial graft, use of antibiotic-soaked graft, and extra-anatomic bypass. However, these methods are not superior to the use of autogenous materials. ${ }^{[6]}$ In the present case, we could reconstruct the EIA and EIV with autogenous materials after resection of the rectosigmoid colon cancer. As far as we know, such kind of reconstruction has not been reported in the English literature.

During other pelvic surgeries, the IIA and IIV are not fully exposed because they are located in the posterior aspect of the rectosigmoid colon. However, since the ipsilateral and contralateral IIA and IIV may be exposed after rectosigmoid colon resection, these vessels may be harvested or mobilized for vessel reconstruction. One of the disadvantages is that the blood supply to the pelvic organs becomes compromised after sacrificing the IIA. Pelvic blood supply is essential for healing of colorectal anastomosis; therefore, surgeons should take into consideration of possibility for decreased blood supply in this situation.

Iliac vein reconstruction is challenging because appropriate graft material is difficult to harvest. Simple ligation of the iliac vein, which is accepted in trauma cases or hemodynamic unstable patients with bleeding, is an option. Several studies proved that vein reconstruction may improve the pa- 
tency of the reconstructed artery, and transient patency of the reconstructed vein may decrease the symptoms and signs of venous hypertension of the involved limb. ${ }^{[7-9]}$ Therefore, vein reconstruction is crucial if there is appropriate graft material. An artificial graft such as PTFE is not suitable for vein reconstruction because of high thrombosis rate; therefore, most surgeons prefer autogenous material. The most commonly used autogenous graft material for vein reconstruction is the great saphenous vein. However, additional time-consuming procedures are mandatory when using a spiral composite graft for iliac vein reconstruction. In cases with rectosigmoid colon cancer resection, the exposed IIV may be used as autogenous graft for reconstruction of the EIA or EIV.

\section{Conclusions}

When major vascular reconstruction is needed after resection of rectosigmoid colon cancer, the IIA and IIV may be used as an autogenous vascular graft material, and this procedure may be used as one of the feasible options for colorectal cancer with external iliac vessel invasion.

\section{CONFlicts OF INTEREST Disclosure}

The authors declare they have no conflict of interest.

\section{REFERENCES}

[1] Abdelsattar ZM, Mathis KL, Colibaseanu DT, et al. Surgery for locally advanced recurrent colorectal cancer involving the aortoiliac axis: Can we achieve R0 resection and long-term survival? Dis Colon Rectum. 2013 Jun; 56(6): 711-6. PMid: 23652744. https://doi.org/10.1097/DCR.0b013e31827dbcb0

[2] Austin KK, Solomon MJ. Pelvic exenteration with en bloc iliac vessel resection for lateral pelvic wall involvement. Dis Colon Rectum 2009 Jul; 52(7): 1223-33. PMid: 19571697. https ://doi .org/10 .1007/DCR. 0b013e3181a73f48

[3] Hashimoto M, Komatsu H, Naruse Y, et al. Resection of paraaortic lymph node metastasis of colon cancer with graft replacement Hepatogastroenterology. 2003 May-Jun; 50(51): 709-10. PMid: 12828066.

[4] Tsarkov PV, Belov YV, Skipenko OG, et al. En bloc resection of abdominal aorta and paraaortic lymph node metastasis of sigmoid cancer. Tech Coloproctol. 2007 Dec; 11(4): 346-9. PMid: 18060362. https://doi.org/10.1007/s10151-007-0379-1

[5] Ueda K, Nagayama H, Narita K, et al. Extended surgery with en bloc resection of the right common iliac vessels for lymph node metastasis of mucinous colon carcinoma: Report of a case. Surg Today. 2001; 31(3): 238-41. PMid: 11318128. https://doi.org/10.1007/s0 05950170176

[6] Dirven M, van der Jagt MF, Barendregt WB, et al. The efficacy of autologous femoropopliteal vein reconstruction for primary aortic and aortic graft infection. Ann Vasc Surg. 2015 Aug; 29(6): 118895. PMid: 26009479. https://doi .org/10.1016/j .avsg. 201 5.03 .043

[7] Phifer TJ, Gerlock AJ Jr, Rich NM, et al. Long-term patency of venous repairs demonstrated by venography. J Trauma. $1985 \mathrm{Apr}$; 25(4): 342-6. PMid: 3989893. https ://doi .org/10.1097/0000 5373-198504000-00012

[8] Phifer TJ, Gerlock AJ Jr, Vekovius WA, et al. Amputation risk factors in concomitant superficial femoral artery and vein injuries. Ann Surg. 1984 Feb; 199(2): 241-3. PMid: 6696539. https: //doi.org/10.1097/00000658-198402000-00018

[9] Rich NM. Principles and indications for primary venous repair. Surgery. 1982 May; 91(5): 492-6. PMid: 7071737. 